\title{
External Quality Assessment on CD4+ T-Cell Count Using in-House Proficiency Testing Panels for CD4 Count Laboratories in Addis Ababa, Ethiopia
}

\author{
Natnael Kidanu Yibalih' ${ }^{1}$, Dawit Wolday ${ }^{2}$, Samuel Kinde ${ }^{3}$, Gebru \\ Mulugeta Weldearegay ${ }^{4}$
}

\footnotetext{
OPEN ACCESS

Citation: Natnaael Kidanu Yibalih, Dawit Wolday, Samuel Kinde, Gebru Mulugeta Weldearegay. External Quality Assessment on CD4+ T-Cell Count Using in-House Proficiency Testing Panels for CD4 Count Laboratories in Addis Ababa, Ethiopia. Ethiop J Health Sci. 2019;29(3):309.

doi:http://dx.doi.org/10.4314/ejhs.v29i3.3

Received: November 7, 2018

Accepted: February 5, 2019

Published: May 1, 2019

Copyright: (C) 2019 Natnaael Kidanu

Yibalih., et al. This is an open access article distributed under the terms of the Creative Commons Attribution License, which permits unrestricted use, distribution, and reproduction in any medium, provided the original author and source are credited.

Funding: Nil

Competing Interests: The authors declare that this manuscript was approved by all authors in its form and that no competing interest exists.

Affiliation and Correspondence:

${ }^{1}$ School of Medicine, Aksum

University, College of Health Science

and Comprehensive Specialized

Hospital, Aksum, Ethiopia

${ }^{2}$ Manager of Medical Biotech

Laboratory, Addis Ababa, Ethiopia

${ }^{3}$ Department of Medical Laboratory

Science, Addis Ababa University,

Addis Ababa, Ethiopia

${ }^{4}$ Department of Medical Laboratory

Science, Addis Ababa University,

Addis Ababa, Ethiopia

*Email:natnaelk1976@gmail.com
}

ABSTRACT

BACKGROUND: CD4+ T-cell count External Quality Assessment program is important for the evaluation of performance of CD4 count laboratories. The aim of this study was to assess the quality of CD4count laboratory performance using in-house Proficiency testing panels that perform routineCD4 counts in Addis Ababa, Ethiopia, 2013/14.

METHODS: Participating laboratories were 20, 23 and 25 in trials 1,2 and 3, respectively. In-house prepared fresh whole blood samples both with "normal" and "low" CD4 values were sent to participating laboratories. Percentage and absolute counts of CD4+ T-lymphocytes were done using their routine procedures. Data were analyzed for each trial including trimmed mean, standard deviation (SD), percent coefficient of variation (\%CV), residual, and standard deviation index (SDI) values for both absolute counts and percentages of CD4+ lymphocytes (\%CD4).

RESULTS: Most participating laboratories produced results that were within $2 S D$ of the mean. Average inter-laboratory precision (trimmed \%CV) was $10.87 \%$ and $5.14 \%$ for CD4 absolute counts and \%CD4, respectively. For normal material, the trimmed mean $\% C V$ was $9.59 \%$ and $3.23 \%$ for CD4 absolute counts and \%CD4, respectively. For low material, the trimmed mean \% CV was $12.15 \%$ and $7.05 \%$ for CD4 absolute counts and \%CD4 respectively. BDFACSCount ${ }^{\mathrm{TM}}$ users showed the best accuracy and precision as evidenced by longitudinal analysis.

CONCLUSION: This study was found to help facilities in early identifying their gaps with regard to their CD4 count performance and in avoiding the challenges encountered during participation in external EQA providers like the high cost, transportation problem, feedback delay and CD4laboratory coverage.

KEYWORDS: External quality assessment, EQA program, CD4 count, in-house proficiency testing panels 


\section{INTRODUCTION}

External quality assessment (EQA) is an evaluation of the performance of a number of laboratories by an outside organization on specially supplied proficiency testing (PT) panels $(1,2)$. EQA scheme monitors the performance of each laboratory over time and identifies those laboratories that require training or corrective action to improve their performance $(3,4)$. One of the best ways for a laboratory to monitor its performance, against both its own requirements and the performance of other laboratories, is to participate regularly in an EQA scheme (5-6).

EQA programs operate through a combination of three approaches that include participation in external PT programs, supervisory site visits by external experts, and retesting a subset of specimens in another competent laboratory or site at a higher level $(8,9)$. There are several well recognized international immune monitoring EQA schemes available for $\mathrm{CD} 4$ testing. In Africa, few laboratories participate in international EQA schemes in comparison with their international counterparts $(10,11)$.

In Africa, the proportion of countries with a national quality assurance (QA) program for HIV has increased from $51 \%$ in 2003 to $61 \%$ in 2007 , but participation was limited mainly to reference laboratories (12). African regional external quality assessment scheme (AFREQAS) (13) was introduced in 2002 in support of this need but this was also mainly limited to reference laboratories $(14,15)$. HIV/AIDS has become a major public health concern, leading the government of Ethiopia to declare a public health emergency in 2002. In 2011, adult HIV/AIDS prevalence in Ethiopia was estimated to be $1.5 \%$ (16).

Accurate absolute CD4+T-cell counts, as well as their percentage values are required for the following purpose:(1) to assess the degree of immune deterioration and rate of progression towards AIDS (defined as a CD4+ lymphocyte count of $<0.2 \times 10^{9} /$ liter or $<14 \%$ ), (2) to group HIV sero-positive patients into cohorts according to their baseline CD4+T-counts before starting treatment(3) to determine the appropriate time for prophylaxis of opportunistic infections, and (4) to monitor the efficacy of antiretroviral and/or interleukin 2 (IL-2) treatment and candidate vaccines(17-20). Therefore, there is clinical need for accurate and precise enumeration of CD4+ Tcells (21-22).

In developing countries, rigorous QA practices for HIV diagnostic and follow-up testing are not routinely followed to ensure reliability of results and a safe work environment. Considering the nature and complexity of EQA to include the need for follow-up supervision and to resolve non-conformances, it is recommended that countries set up functional EQA program to be coordinated at national level with the capacity to either produce or procure PT panels to distribute to other laboratories (23-25).

According to Brando et al, EQA programs for clinical cell analysis should not be doomed to remain monopolized by a few major providers worldwide even if there are challenges. One of the key challenges in running PT program has been that of the cost and distribution to include the issues of maintaining cold chain, packaging and shipment with maximum safety to laboratory staff and people involved in the shipment exercise $(25,26)$.

In Ethiopia, specifically in Addis Ababa, there is extensive expansion of public and private ART sites for the treatment and monitoring of HIV infected patients. The two most commonly available and used flow cytometry machines in Ethiopia for ART initiation and monitoring areBD FACSCount, which is available almost in all ART laboratories and BD FACSCalibur that is available at a referral level and some private laboratories (27).

In Ethiopia, different ART CD4 count laboratories participate in EQA programs through Quality Assessment Scheme International (QASI) (that is coordinated by the Ethiopian Health and Nutrition Research Institute (EHNRI)) and/or AFREQAS that send-out lyophilized materials. Since the imported lyophilized panels need reconstitution upon supplier's instructions, this process reduces the commutability of the materials and feedback takes long time (above six months) after result submission. Other problems associated with participation include the high cost of EQA schemes, logistical problems with sample transport to testing sites, and lack of infrastructure

DOI: http://dx.doi.org/10.4314/ejhs.v29i3.3 
and computers to enter data onto websites for submission of results. In addition, international EQA schemes are frequently commercial investments, and their focus may not necessarily be the development of quality laboratory capacity building in resource-poor settings $(1,11,13)$.

Thus, the objective of this study was to assess the quality of CD4 + count laboratory performance using in-house PT panels in Addis Ababa, Ethiopia.

\section{MATERIALS AND METHODS}

Study participants: Public, non-governmental organization, and private CD4 count laboratories were included in the study based on their provision of routine CD4 testing voluntarily. The study was conducted in Addis Ababa, Ethiopia, from December 2013 to July 2014 after ethical clearance was obtained from the School of Medical Laboratory Sciences, College of Health Sciences, Addis Ababa University and Addis Ababa City Administration Health Bureau. Relevant data like flow cytometer and antibody panel used, internal quality control and pipetting method used, pipette calibration, bead product used, and work load of each laboratories were gathered by using preliminary structured questionnaire. Three trials were carried out, and out of 27 laboratories, 20, 23 and 25 were participated in trials 1, 2 and 3, respectively.

Preparation of EQA material for shipment: The preparation was done at Medical Biotech Laboratory (MBL). MBL participates in CD4 EQA scheme with digital PT Canada (organized through EHNRI) and in AFREQAS (organized through National Health Laboratory Service, Johannesburg, South Africa) quarterly and every two monthly respectively with excellent performance. In-house PT material was prepared by drawing predetermined CD4 value and HBsAg, HCV, HIV-1 and HIV-2 negative fresh whole blood obtained from one donor in to $4 \mathrm{ml}$ K3-EDTA BD vacutainer tubes (8-12 tubes). The collected blood was mixed thoroughly in automated hematology-mixer machine to insure homogeneity of the materials. Once mixed, it was then pooled into a large sterile container with 50 $\mathrm{ml}$ volume capacity (Costar, Corning, Inc., Corning, NY) and gently mixed again to ensure uniformity of pooled samples. The above blood was separated into two samples (sample-A and B) to prepare the normal (sample-A) and low (sample-B) EQA materials.

Normal EQA material was prepared in such a way that it coincides with Ethiopian CD4 Tcellcounts $(753 \pm 227$ cells $/ \mu 1$ for adult male) (28). Low EQA material was prepared by continuous dilution of sample-B using phosphate buffer saline (PBS) until desirable low CD4 count was attained (100-300 cells $/ \mu 1$ range). Similar preparation of fresh whole blood for CD4 EQ Ascheme was reported by Kunkl et al, in 2002 (29). Normal and low materials were packed according to WHO guidelines before dispatch time $(30-33)$. Each batch of EQA blood was evaluated to ensure that immuno-phenotypic characteristics have been maintained during handling and transportation.

Dispatch of CD4 EQA material: All shipments included in-house prepared PT material and its handling and running instruction sheets, data report form with unique participant identifier, clear instructions of the date of submission deadline, and safety information. It was personally delivered by the principal investigator at the same day of blood collection to each participant throughout the 3 trials. On the dispatch dates, the material was sent in plastic transport bag box to maintain optimal temperature by avoiding direct sunlight (at ambient temperature $\left(18-22^{\circ}\right)$ ). All participants received their samples within 1-8 hours of EQA material dispense. All communications were mainly based on telephone and personal contact before and during all the dispatch dates.

Statistical analysis of trial data: Microsoft Excel $^{\mathrm{TM}}$, sigma plot version 12.3, and Graph $\operatorname{Pad} \AA$ Prism version 5, were used to capture data and to generate statistics, tables, graphs, letters and feedback to all participating laboratories.

Each participant's data was analyzed according to international practice $(5,6,10,13)$. On receipt of all participant data for each trial, CD4 absolute counts and CD4\% of Lymphocyte values were entered into Microsoft Excel ${ }^{\mathrm{TM}}$. The mean, standard deviation $(\mathrm{SD}=[\sqrt{ } \Sigma(\mathrm{X} 1-\mathrm{X}) 2 /(\mathrm{n}-1)])$, and coefficient of variation $(\% \mathrm{CV}=\mathrm{SD} /$ Mean $\mathrm{x} 100)$ were calculated automatically by the

DOI: http://dx.doi.org/10.4314/ejhs.v29i3.3 
Microsoft Excel ${ }^{\mathrm{TM}}$, and a \pm 2SD limit determined for both CD4 parameters (untrimmed pooled data). Results that fell outside the \pm 2 SD limit were identified and removed as outliers and pool data re-analyzed to calculate pooled trimmed mean, pooled trimmed SD, and pooled trimmed $\%$ CV. Each participant's "Residual" expressed in CD4 cells/ $\mu 1$ or CD4\% (equal to participant's submitted CD4 absolute or CD4\% result minus the trimmed pool mean result) and the standard deviation index (SDI) (equal to participant's "Residual" divided by SD of the trimmed mean, expressed as a ratio) were calculated and compared to the pooled trimmed results to indicate bias(31). The overall participation response rates (RR) were calculated as the (number of submitted results) divided by (the number of shipped samples). Non-return rates (NR) were calculated as the (number of laboratories that did not return results) divided by the (number of shipped samples). Percentage of outlying results or "Outlier Rate" (OR) was calculated as (number of sites with $\mathrm{SD}>2.0$ of the trimmed pool mean) divided by the (number of submitted results). All rates were expressed as percentages.

Participants were informed about the procedure of feedback and identifying problems to assist laboratories with trouble shooting. Those participants whose results were outside the $\pm 2 \mathrm{SD}$ of the trimmed pool mean were contacted immediately to effect corrective action timely and were not allowed to resubmit corrected results. This was based on advice on different technologies and guidelines for equipment maintenance and regular service of instruments. Participant laboratories were also advised to use a Levey-Jennings wall chart to plot and monitor their performance over time.

Assessment of longitudinal performance of individual participants: Individual SDI performance of laboratories was monitored longitudinally by graphically representing the SDI values versus trial number on a radar graph $(13,21)$. These radar plots enabled visual representation of the accuracy and precision of CD4 absolute counts and CD4\%of Lymphocytes for individual participants $(13,20)$.
Statistical analysis of overall precision of BD FACSCount ${ }^{\text {TM }}$ users: For each trial, submitted results for both absolute counts and CD4\% of Lymphocytes was pooled and mean, SD and $\% \mathrm{CV}$ calculated to assess their accuracy and precision. The Longitudinal precision was analyzed ( $\% \mathrm{CV}$ over the 3 trials) for both absolute counts and CD4\% of Lymphocytes. These results were plotted on scatter plots using Graph Pad Software. To assess the longitudinal accuracy and precision of BD FACSCount ${ }^{\mathrm{TM}}$ users, the standardized individual laboratory SDI values were pooled across the 3 trials. Thus a mean (SDI) was calculated per trial, and Radar plots were used to show the mean of the pooled SDI values plotted trial by trial. Also, it was plotted in Gaussian distribution plot. In this pooled SDI analysis, the calculated mean of the pooled SDI reflects longitudinal accuracy. The SD of the mean of the SDI reflects longitudinal precision. In this analysis, ideal accuracy mean (SDI) values should be expected to be 0.0 with an expected range of -1 to +1 . Ideal precision $\mathrm{SD}$ (Mean SDI) values should be expected to be 1.0 with an expected range of 0.0 to 1.0.

\section{RESULTS}

Participant laboratories: The number of participants in the study increased from 20 to 25 laboratories (almost all laboratories in Addis Ababa) by trial 3, representing an average monthly growth rate of $11.9 \%$. The study issued 3 trials, equating to 136 in-house PT panels of which 68were "normal" and 68 "low" CD4 value materials. The response rate was $100 \%$.

Performance of all participant laboratories: Over the 3 trials, participant laboratories showed an average between laboratory precision/reproducibility (upper acceptable limit is $10 \% \mathrm{CV}$ ) for absolute CD4 counting of $10.87 \%$ $\mathrm{CV}$, ranging between $7.88 \%$ (Trial $1 \mathrm{~A}$ ) to $15.49 \%$ (Trial 3B). The trimmed mean between laboratory precision for CD4\% of lymphocytes was $5.14 \%$ $\mathrm{CV}$, ranging between $2.25 \%$ (Trial $1 \mathrm{~A}$ ) to $10.44 \%$ (Trial 2B).

For normal CD4 value material, the trimmed mean $\% \mathrm{CV}$ was $9.59 \%$ and $3.23 \%$ for CD4absolute counts and CD4\% of Lymphocytes, respectively. For low CD4 value material, the

DOI: http://dx.doi.org/10.4314/ejhs.v29i3.3 
trimmed mean \% $\mathrm{CV}$ for $\mathrm{CD} 4$ absolute counts and CD4\% of lymphocytes was $12.15 \%$ and $7.05 \%$, respectively. Overall, the participating laboratories showed relatively poorer precision $(\% \mathrm{CV})$ and accuracy with "low" material than with "normal" material for both CD4 absolute counts and CD $4 \%$ of lymphocytes. In trials 1 and 2 only, 2 results (from trial 2) were out of \pm 2 SD with the normal material, but 4 results (three from trial 2 and one from trial 1) were out of \pm 2 SD from low material. In the same manner, one result from normal material and 2 results from low material were out of \pm 2SD in trial 3 as well.

Participant performance was characterized as excellent and good based on the number of outliers and non-submissions. Excellent performers had neither outliers nor nonsubmissions during their time of participation. Good performers had 2 or less outliers/nonsubmissions for the duration of their participation. A similar grouping criterion was used elsewhere $(1,13)$.

Overall, $81.48 \%$ of the participants reporting CD4 absolute counts and $76.92 \%$ of participants reporting CD4\% of lymphocyte showed excellent serial continued performance across 3 trials. Here, the methodologies used comprised of BD FACSCount ${ }^{\mathrm{TM}} \quad$ users, Pima $^{\mathrm{TM}} \mathrm{CD} 4$, BDFACSCalibur ${ }^{\mathrm{TM}}$, and a single laboratory using PartecCy Flow counter test. A second group of users defined as good performers comprised further $18.52 \%$ of participants reporting CD4absolute counts and $23.08 \%$ participants reporting $\mathrm{CD} 4 \%$ of lymphocyte. The methodologies here are mainly Pima TM CD4. In the group with "good performers", $40 \%$ of them showed improvement in the third trial as opposed to consecutive outliers.

Precision of laboratories using the same CD4 methodology: During the three trials, BD FACSCount ${ }^{\mathrm{TM}}$, $\quad \mathrm{Pima}^{\mathrm{TM}} \mathrm{CD} 4, \quad$ BD FACSCalibur ${ }^{\mathrm{TM}}$, and PartecCy Flow counter were performed as a single platform test but except BD
FACSCount ${ }^{\mathrm{TM}}$, their numbers were insufficient (less than 10) for statistical analysis as a group. As a result, they were excluded from group comparison, but their performance was assessed together with all participants. The numbers of BD FACSCount $^{\mathrm{TM}}$, BD FACSCalibur ${ }^{\mathrm{TM}}$ and Pima ${ }^{\mathrm{TM}}$ CD4 users were 17, 4 and 4, respectively by the end of the third trial, and Partec ${ }^{\circledR}$ methodology was a single participant in the first two trials. The average number of BD FACSCount ${ }^{\mathrm{TM}}$ users was 16 laboratories. In trial 2, $13.78 \% \mathrm{CV}$ was recorded which was relatively poor, which was due to a single outlier where one participant reported a CD4 absolute count of 386 cells $/ \mu 1$ for low material. On removing this outlying result, (Grubbs' test of outlier applied), the overall trimmed mean for all technology users was 265.82 with a \%CV reduced by $3.12 \%$ CV.BD FACSCount ${ }^{\mathrm{TM}}$ methodology.

Across the 3 trials, performance of $\mathrm{BD}$ FACSCount $^{\mathrm{TM}}$ users (comprising 72.67\% of participants) for CD4 absolute counts showed consistently good performance. The overall mean precision $(\% \mathrm{CV})$ was $9.13 \%$ and $5.15 \%$ for absolute CD4 counts and CD4\% of lymphocytes, respectively. For normal CD4 value material, a trimmed mean $\% \mathrm{CV}$ of $8.58 \%$ and $3.07 \%$ was recorded for absolute CD4 count and CD4\% of lymphocytes, respectively. For low CD4 value material, the trimmed mean $\% \mathrm{CV}$ was $9.67 \%$ and $7.22 \%$ for absolute CD4 count and CD4\% of lymphocytes, respectively.

To assess longitudinal accuracy and precision, the standardized individual laboratory SDI values were pooled across 3 trials and a mean (SDI) was calculated per trial (Figures 1-3). Pooled SDI data reflecting longitudinal accuracy and precision across 3 trials showed that laboratories using BD FACSCount ${ }^{\mathrm{TM}}$ could consistently generate accurate and precise absoluteCD4 counts irrespective of whether normal or low CD4 value material was used (Figure 2,3). 


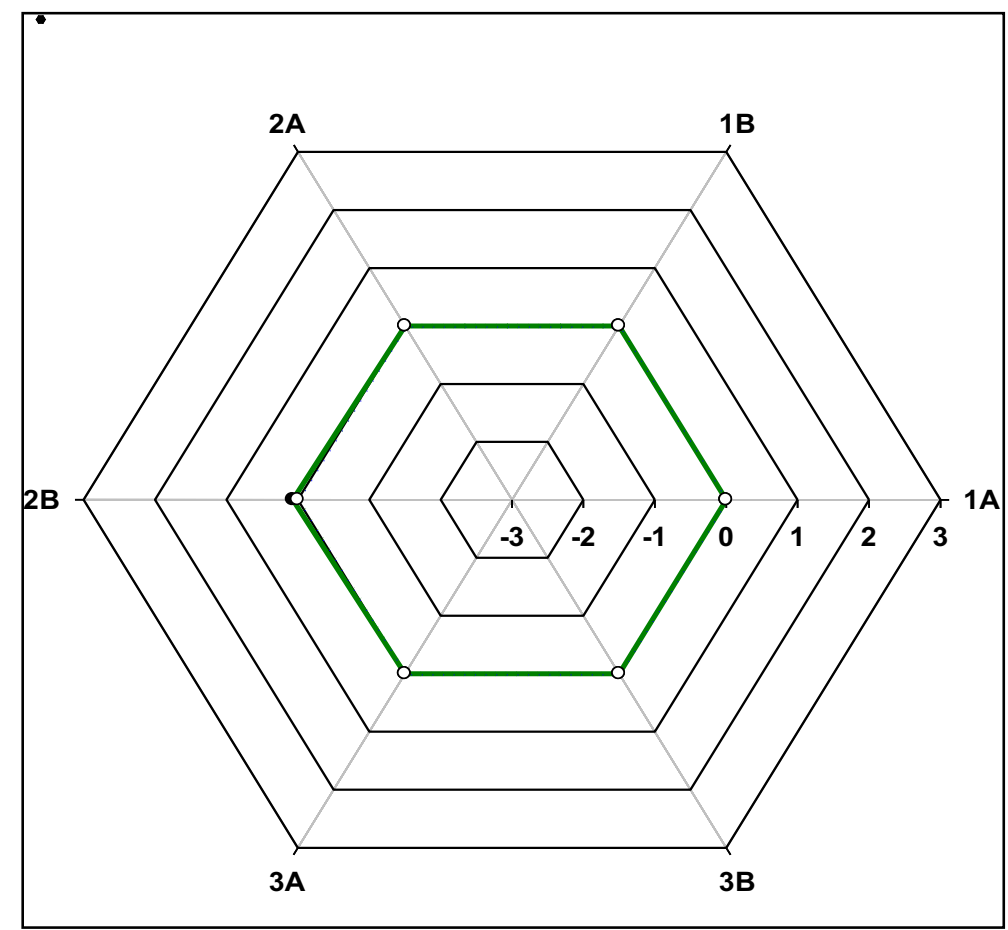

Figure 1: Radar plots illustrating the longitudinal performance (SDI) of BD FACSCount ${ }^{\mathrm{TM}}$ methodology across the three trials

In the above figure, the $\mathrm{X}$-Axis reflects SDI performance on a scale of -3 to +3 where acceptable range is -2.0 to +2.0 . Y-Axis indicates the consecutive trial. SDI values were plotted for both the absolute CD4 count (Green line) and CD4\% of lymphocyte values (totally obscured by the target line because they have same value i.e. zero). As a result; it is hidden.

On low CD4 value material, longitudinal follow-up showed slightly poorer accuracy (mean
SDI) and precision than with normal CD4 value material. This, however, did not impact on the overall good performance of this methodology on the study. Only one trial showed relatively poorer precision (Trial 2). This was due to one outlier where one participant reported CD4\%lymphocyte (Trial 2B) of 24.92\% (pool mean 32.62).

DOI: http://dx.doi.org/10.4314/ejhs.v29i3.3 
BD FACSCount: Absolute CD4

Accuracy Normal Value Material

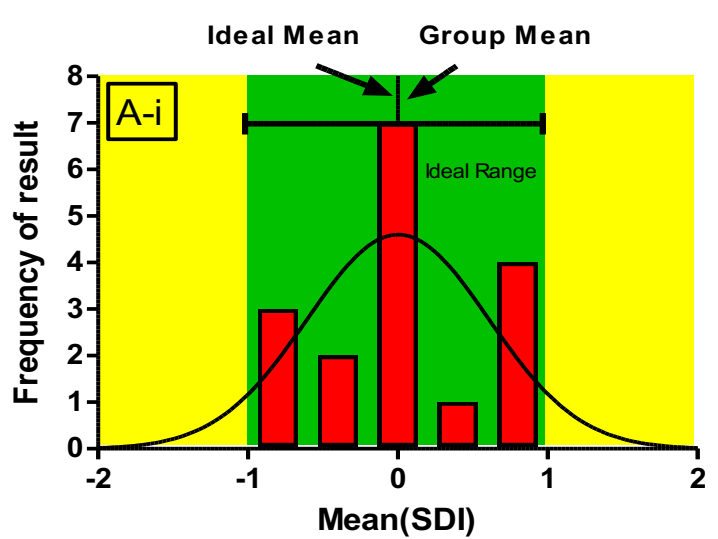

\section{Low Value Material}

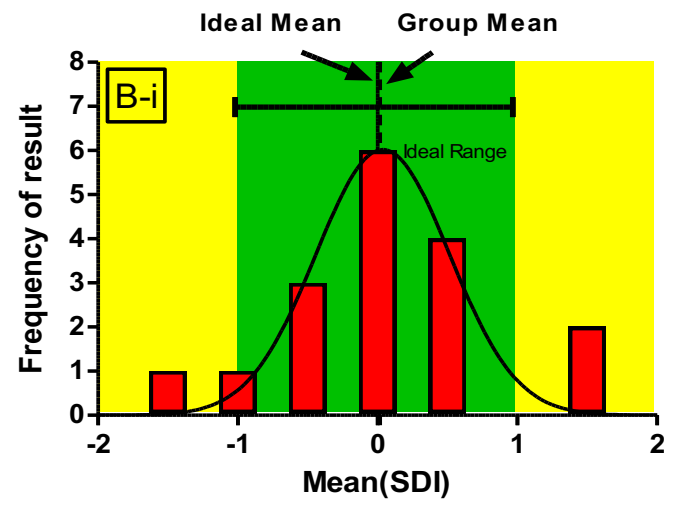

Precision
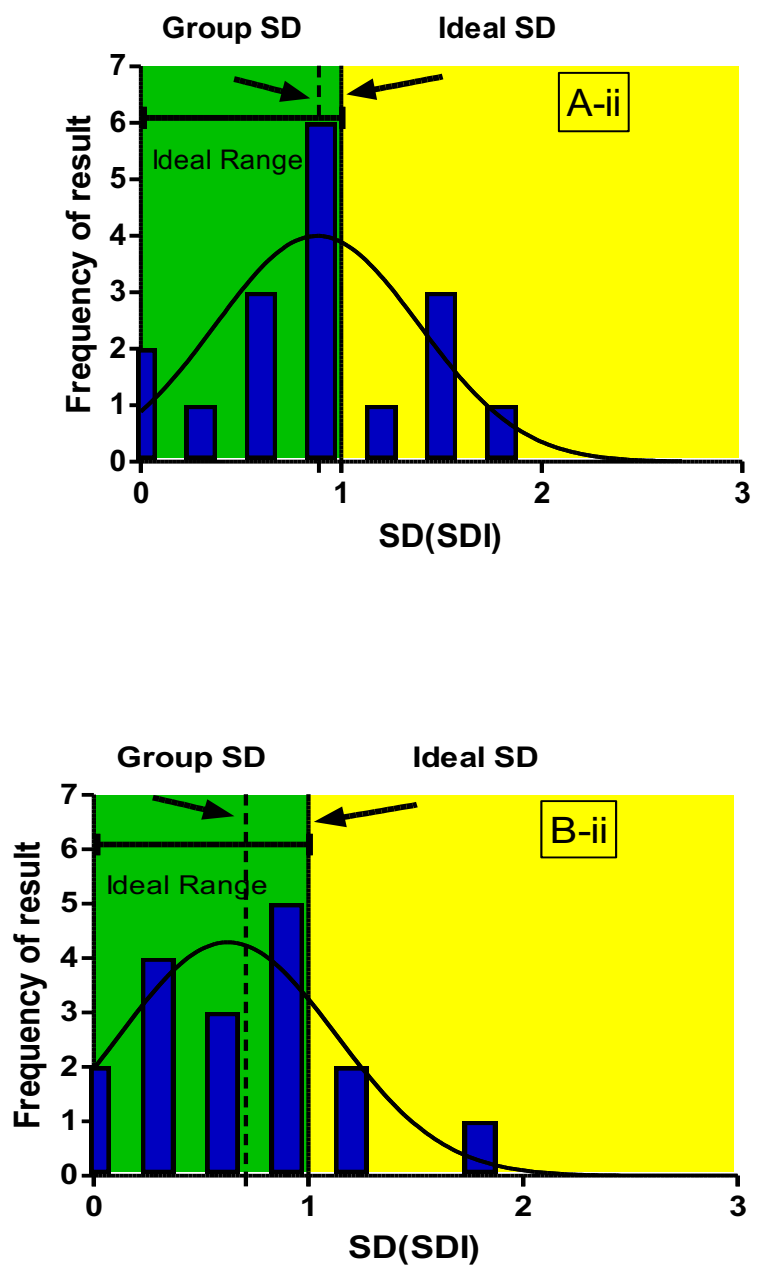

Figure 2: Gaussian distribution curves showing longitudinal accuracy (mean SDI, Ai and B i) and precision (SD of mean SDI), Aii and B ii) for BDS FACSCount ${ }^{\mathrm{TM}}$ users for CD4 absolute counts.

In figure 2, Green areas represent the acceptable limits for both Mean SDI and SD of (mean SDI) values, while yellow areas indicate values outside the acceptable limits. Ideal values for both mean SDI (0) and SD of (mean SDI) (1) are indicated. 
BD FACSCount: CD4\% of Lymphocyte

Accuracy

Normal Value Material

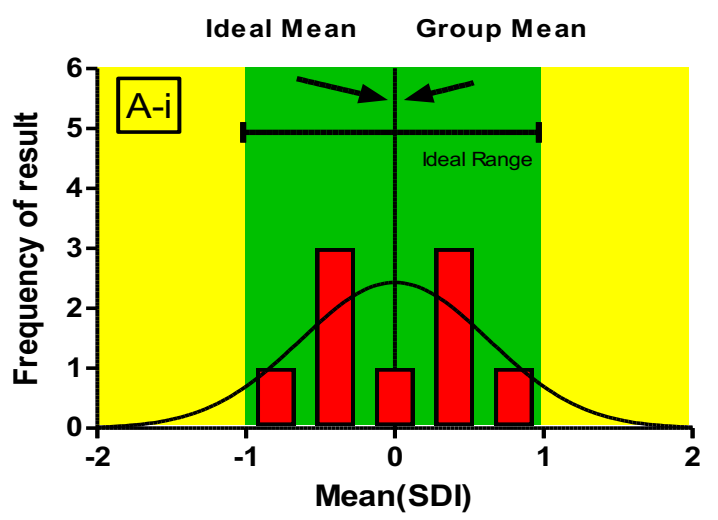

Low Value Material

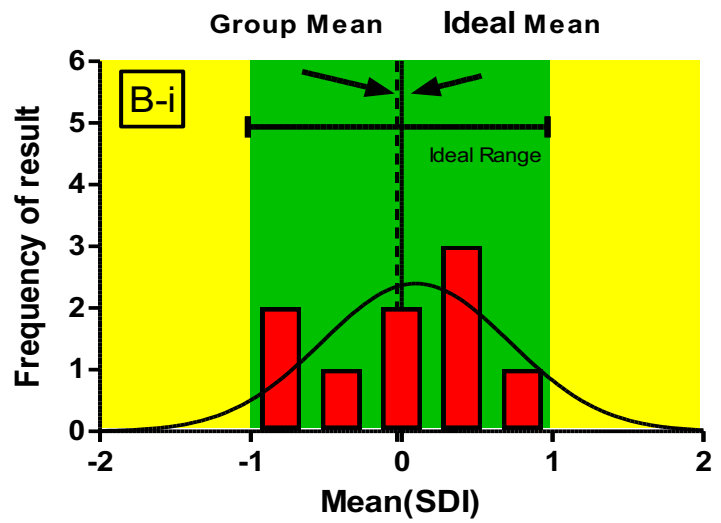

\section{Precision}
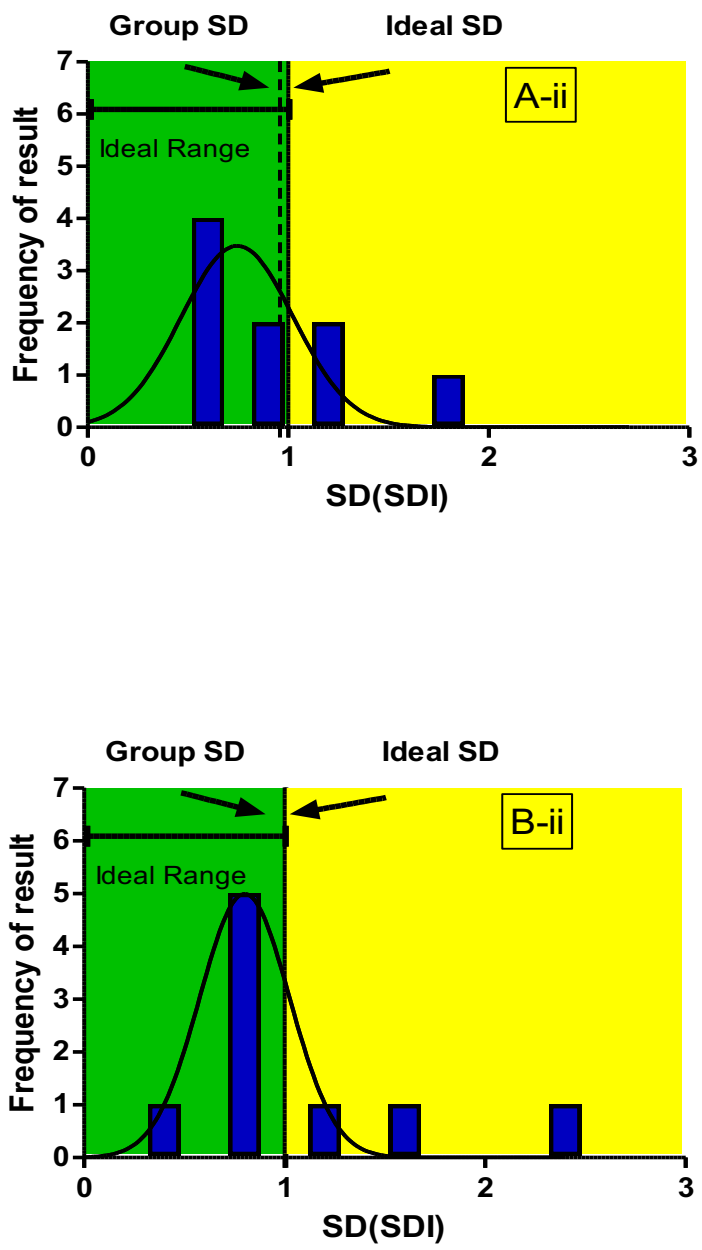

Figure 3: Gaussian distribution curves showing longitudinal accuracy (mean SDI, Ai and Bi) andprecision (SD of mean SDI), Aii and B ii) for BD FACSCount ${ }^{\mathrm{TM}}$ users for CD4\% of lymphocyte

In figure 3, greenare as represent the acceptable limits for both Mean SDI and SD of (mean SDI) values, while yellow areas indicate values outside the acceptable limits. Ideal values for both mean SDI (0) and SD of (mean SDI)(1) are indicated.

Investigation of laboratories with outlying results: An outlier was defined as a submitted result falling outside of $\pm 2 \mathrm{SD}$ of the trimmed pool mean. The percentage outlier rate (OR) of absolute CD4 counts and CD4\% of lymphocytes for all trials were $4.3 \%$ and $4.1 \%$ respectively.
The percentage OR of normal CD4 EQA material was $1.4 \%$ and $5.3 \%$ for absolute counts and CD4\% of Lymphocyte respectively and that of low CD4 EQA material was $7.2 \%$ and $2.8 \%$ for absolute counts and CD4\% of Lymphocyte respectively. OverallBD FACSCount ${ }^{\mathrm{TM}}$ and BD FACSCalibur users showed the least outliers across 3 trials with an average of $<5 \%$ and $<7 \%$ respectively. The Pima ${ }^{\mathrm{TM}} \mathrm{CD} 4$ user group showed higher outliers across the 3 trials with an average of $<17 \%$. The single Partec ${ }^{\circledR}$ user laboratory had no outliers and non-submission of results across the first 2 trials. 
Effects of feedback for remedial action: Feedback was individualized and remedial input given was specific to the problem of the CD4method used by that site. These include appropriate feedback and advice on corrective action. Outliers on CD4 absolute counts and CD4\% of Lymphocytes were noted on Trial 1 and the laboratories were subsequently contacted to investigate the inaccuracy and imprecision of pipetting through the validation of pipettes based on their respective methods user's guide. With corrective action feedback to these laboratories, no outliers reported consistently for the last two subsequent trials.

\section{DISCUSSION}

During the 3 trials, the overall participation rate was excellent with average $93.1 \%$. The average response rate was $100 \%$. Overall, reasons for $100 \%$ response rate were due to consistent information exchange between the study coordinator and managers of participant laboratories.

The other $6.9 \%$ of potential laboratories were not participated. This was due to poorly developed flowcytometry maintenance, lack of BD FACSCount Reagents, lack of supplier and vendor availability for support and maintenance of equipment, unreliable power supplies, poorly developed custom clearance and infrastructure.

The average precision of all participant laboratories across the 3 trials was 10.87 and $5.14 \% \mathrm{CV}$ for absolute CD4 counts and CD4\% of Lymphocytes, respectively. This performance is better than the overall precision $(\% \mathrm{CV})$ reported on the AFREQA scheme across 20 trials of $11.9 \mathrm{CV} \%$ for absolute CD4 counts and 10.7 CV\% for CD4\% of Lymphocytes (13). This can be explained by the fact that in-house PT panels were shipped within the same day of preparation to participants but in AFREQAS transportation of EQA materials was taken longer time. As a result, the commutability of materials can be reduced through time and temperature fluctuation. In AFREQAS, the huge numbers of participants used single and dual platform technology that may increase the $\% \mathrm{CV}$, but in this study, all participants were used only single platform technology.
Another study done by Lopez et al reported similar improved precision mean $\mathrm{CV}$ of less than $10 \%$ (7). It is a little bit higher than the overall precision $\% \mathrm{CV}$ reported on the EQA Program in Thailand of less than $8 \%$ for CD4 T-lymphocytes (20). According to the information obtained from preliminary structured questionnaire and data report form, most of the participants do not calibrate their pipettes and in a few laboratories, mismatched reagent lot number (ID) and control run reagent lot number (ID) was found. This is probably the reason for the increased $\% \mathrm{CV}$. With the "normal" and "low" CD4 value material, similar findings were reported in other studies $(13,18)$, with overall slightly poorer precision with "low" count material than "normal" count material amongst participants.

Overall accuracy and precision of BD FACSCount ${ }^{\mathrm{TM}}$ users was excellent as evidenced by the tight Mean SDI and narrow SD (of the Mean SDI) noted in the longitudinal analysis (Figure 2,3). This excellent performance was maintained irrespective of whether "normal" or "low" material was used and a mean $\% \mathrm{CV}$ of less than $10 \% \mathrm{CV}$ was recorded for both normal and lowCD4 absolute counts and CD4\% of lymphocytes across 3 trials. A similar study was conducted in Thailand $(13,20)$. One error, common to several BD FACSCount ${ }^{\mathrm{TM}}$ users, was related to incorrect pipetting and use of poorly calibrated pipettes, also reported elsewhere $(7,13)$.

Overall, the performance of the CD4 laboratories in Addis Ababa is similar to that reported elsewhere $(7,13,15,20)$. However, few of the CD4 methodologies, specifically one Pima ${ }^{\mathrm{TM}} \mathrm{CD} 4$, showed inconsistent results between trials. The probable reason may be the difficulty to evaluate as group users due to their small number. In addition, these technologies were new and three of them were incorporated at the second trial of the study and were allowed to give service for patients as they purchased without in-house validation. Other difficulties with new coming methods are: laboratory technicians were not trained about the usage and maintenance of the machines rather deputies of the seller (vendor) company install it in the laboratory and show

DOI: http://dx.doi.org/10.4314/ejhs.v29i3.3 
them how they perform at the first time of purchasing.

The findings of this study showed that those laboratories which submitted results with outliers in trial 1 were consistently reported with no outliers in the subsequent trials after appropriate feedback and advice was given. Thus, it is reasoned that the longer the laboratories participating in the EQA trial, the better the performance of these laboratories. There are some limitations to this study. Firstly, unlike that of other CD4 EQA schemes done elsewhere with multiple numbers of trials, this study was done in a short period of time. This was due to lack of sponsors since it needed a huge amount of budget for its continuity and sustainability. Secondly, corrective action feedback was solely based on advice and provision of guidelines, but training was not given even if many requests were reported from participating laboratories. This was due to limited inputs and lack of sponsorship.

To conclude, the pilot-feasibility of preparation of in-house PT panel for CD4 enumeration was assessed and found to be satisfactory. Our study was helpful in early identifying of participants' gaps with regard to their CD4 count performance. This study may be considered as a significant advancement for CD4count laboratories in Addis Ababa and at national level for its use as a baseline, since no such study was available previously. Above all, this study is helpful to avoid external dependency on CD4 EQA. This is because participation in externally provided EQA scheme is difficult in terms of cost, transportation, coverage of all laboratories, on-time feedback and communication. As a result, this study has been widely accepted by participant laboratories.

In Ethiopia, there is no established national CD4 EQA scheme. As a result, it is best to expand and continue this study as a program using in-house prepared PT panels at national level in a sustainable way.

\section{ACKNOWLEDGEMENTS}

The study was supported financially by Addis Ababa University. Special thanks go to the research assistants who were involved in data collection.

\section{REFERENCES}

1. Aggett H. The impact of a CD4 external quality assessment programme for Southern Africa and Africa: Faculty of Health Sciences, University of Witwatersrand; 2009.

2. Stevens W. Good clinical laboratory practice (GCLP): the need for a hybrid of good laboratory practice and good clinical practice guidelines/standards for medical testing laboratories conducting clinical trials in developing countries. Qual Assur. 2003;10(2):83-9.

3. Barnett D, Granger V, Whitby L, Storie I, Reilly JT. Absolute CD4+ T-lymphocyte andCD34+ stem cell counts by singleplatform flow cytometry: the way forward. $\mathrm{Br}$ J Haematol. 1999;106(4):1059-62.

4. Brando B, Sommaruga E. Nationwide quality control trial on lymphocyte immunophenotyping and flow cytometer performance in Italy. Cytometry. 1993;14(3):294-306.

5. Gelman R, Cheng SC, Kidd P, Waxdal M, Kagan J. Assessment of the effects of instrumentation, monoclonal antibody, and fluorochrome on flow cytometric immunophenotyping: a report based on 2 years of the NIAID DAIDS flow cytometry quality assessment program. Clin Immunol Immunopathol. 1993; 66(2):150.

6. Libeer JC, Baadenhuijsen H, Fraser CG, Petersen PH, Ricos C, Stockl D, et al. Characterization and classification of external quality assessment schemes (EQA)according to objectives such as evaluation of method and participant bias and standard deviation. External Quality Assessment (EQA) Working Group A on Analytical Goalsin Laboratory Medicine. Eur J Clin Chem Clin Biochem. 1996; 34(8):665.

7. Lopez A, Caragol I, Candeias J, Villamor N, Echaniz P, Ortuno F, et al. Enumeration ofCD4 (+) Tcells in the peripheral blood of HIV-infected patients: an inter-laboratory study of the FACSCount system. Cytometry. 1999; 38(5):231-7.

8. Laessig RH, Ehrmeyer SS, Leinweber JE. Intralaboratory performance requirements

DOI: http://dx.doi.org/10.4314/ejhs.v29i3.3 
necessary to pass proficiency testing: CAP1990 vs CLIA-1967 (March 14, 1990) formats compared. Clin Chem. 1992; 38(6):895-903.

9. Lanphear BJ, Burmeister BJ, Ehrmeyer SS, Laessig RH, Hassemer DJ. Review of actual proficiency testing performance under CLIA '67(March 14, 1990) rules: perspective from the first year's data. Clin Chem. 1992; 38(7):1254-9; discussion 68-72.

10. Whitby L, Granger V, Storie I, Good fellow K, Sawle A, Reilly JT, et al. Quality control of CD4+ T lymphocyte enumeration: results from the last 9 years of the United Kingdom National External Quality Assessment Scheme for Immune Monitoring (1993-2001). Cytometry. 2002;50(2):102-10.

11. Mandy F, Bergeron M, Houle G, Bradley J, Fahey J. Impact of the international program for Quality Assessment and Standardization for Immunological Measures Relevant to HIV/AIDS: QASI. Cytometry. 2002; 50(2):111-6.

12. World health organization/Regional Office for Africa. HIV and AIDS laboratory capacity where are we? : Overview of laboratory capacity in Africa 2005-2007.Brazzaville, Republic of Congo; 2010.

13. Glencross DK, Aggett HM, Stevens WS, Mandy F. African regional external quality assessment for CD4 T-cell enumeration: development, outcomes, and performance of laboratories. Cytometry part B: Clin Cytom. 2008; 74 (S1):S69-S79.

14. Vercauteren G, Aggett HM, Mandy FF, Glencross DK. Impact of the WHO External Quality Assessment (EQA) program on the laboratory performance for CD4enumeration for monitoring of ARV therapy in resource limited settings. XVI International AIDS Conference: Toronto, Canada; 2006.

15. Glencross DK, Aggett HM, Stevens W, Bergeron M, Houle G, Mandy FF. Improved between laboratory performance of South African NHLS laboratories using PLG CD4methodology for the national antiretroviral treatment program. XVI International AIDS Conference: Toronto, Canada; 2006.
16. Central Statistical Agency of Ethiopia and ICF International. Ethiopia Demographic and Health Survey 2011. Addis Ababa, Ethiopia and Calverton, Maryland, USA: Central Statistical Agency and ICF International. 2012.

17. Huang KH, Loutfy MR, Tsoukas CM, Bernard NF. Immune correlates of CD4 decline in HIV-infected patients experiencing virologic failure before undergoing treatment interruption. BMC Infect Dis. 2008;8(1):59.

18. Reilly JT, Barnett D. UK NEQAS for leucocyte immunophenotyping: the first 10 years.J clinpathol. 2001;54(7):508-11.

19. Barnett D, Walker B, Landay A, Denny TN. CD4 Immunophenotyping in HIV infection. Nat Rev Microbiol. 2008;6(S11):S7-S15.

20. Pobkeeree V, Lerdwana S, Siangphoe U, Noulsri E, Polsrila K, Nookhai S, et al.External Quality Assessment Program on CD4+ T-Lymphocyte Counts for Persons with HIV/AIDS in Thailand: History and Accomplishments. Asian Pacific Journal of Allergy and Immunology. 2009;27(4):225-32.

21. Stevens W, Gelman R, Glencross DK, Scott LE, Crowe SM, Spira T. Evaluating newCD4 enumeration technologies for resourceconstrained countries. Nat Rev Microbiol.2008; 6(S11):S29-S38.

22. Glencross DK, Janossy G, Coetzee LM, Lawrie D, Aggett HM, Scott LE, et al. Largescale affordable PanLeucogated CD4+ testing with proactive internal and external quality assessment: In support of the South African national comprehensive care, treatment and management program for HIV and AIDS. Cytometry part B: Clin Cytometry. 2008;74(S1):S40-S51.

23. Westerman LE, Kohatsu L, Ortiz A, McClain B, Kaplan J, Spira T, et al. A Quality Management Systems Approach for CD4 Testing in Resource-Poor Settings. Am J clinpathol. 2010;134(4):556-67.

24. Jiang Y, Qiu M, Zhang G, Xing W, Xiao Y, Pan $P$, et al. Quality assurance in the HIV/AIDS laboratory network of China. International Journal of Epidemiology.2010;39: ii72-ii78. 
25. Alemnji G, Nkengasong JN, Parekh BS. HIV testing in developing countries: What is required?. Indian J Med Res. 2011; 134(6):779-786.

26. Brando B, Gatti A, Chianese R, Gratama JW. Twenty years of external quality assurance in clinical cell analysis-A tribute to JeanJean-Luc D'Hautcourt. Cytometry Part B: Clinical Cytometry. 2007;72(1):2-7.

27. Family Health International (FHI)-Ethiopia/ Addis Ababa City Administration Health Bureau (AACAHB). Addis Ababa HIV care and support service assessment For Ethiopia. 2002.

28. Tsegaye A, Messele T, Tilahun T, Hailu E, Sahlu T, Doorly R, et al. Immunohematological reference ranges for adult Ethiopians. Clin Diagn Lab Immunol.1999;6(3):410-4.

29. Kunkl A, Risso D, Terranova MP, Girotto M, Brando B, Mortara L, et al. Grading of laboratories on $\mathrm{CD} 4+$ T-lymphocyte evaluations based on acceptable data boundaries defined by the measurement error. Cytometry. 2002;50(2):117.

30. World Health Organization/Emerging and other Communicable Diseases, Surveillance and Control (WHO/EMC/97.3). Guidelines for the safe transport of infectious substances and diagnostic specimens. 1997.

31. Healy MJ. Outliers in clinical chemistry quality-control schemes. Clin Chem. 1979;25(5):675-7.

32. Deom A, El Aouad R, Heuck CC, Kumari S, Lewis SS, Uldall A, et al. WHO/DIL/LAB/99.2: Requirements and guidance for external quality assessment schemes for health laboratories. World health organization; 1999.

33. World Health Organization/SEA. New Delhi. Laboratory guide-lines for enumerating CD4 T-lymphocytes in the context of HIV/AIDS. 2007. 